\title{
Evidence and Controversies in the Use of Post-Mastectomy Radiation
}

\author{
Shaneli A. Fernando, MD; ${ }^{a}$ and Stephen B. Edge, MD, ${ }^{\text {b, }}$ Buffalo, New York
}

\begin{abstract}
Key Words
Breast cancer, post-mastectomy radiation, locoregional failure, chemotherapy, mastectomy, lymph node, NCCN Breast Cancer Clinical Practice Guidelines in Oncology
\end{abstract}

\begin{abstract}
Radiation after mastectomy is recommended for women with positive nodes, larger tumors, or positive margins. In addition to its role in reducing the risk for locoregional recurrence, it has an additive effect to the survival benefit seen with adjuvant systemic therapy. This article reviews the role of post-mastectomy radiation (PMRT) based on the recommendations in the 2007 NCCN Breast Cancer Clinical Practice Guidelines in Oncology and addresses the risk factors for recurrence after mastectomy. The data supporting both improvement in locoregional failure and survival are reviewed in detail. This article also discusses controversial areas in PMRT, including regional nodal radiation in women with 1 to 3 positive lymph nodes, PMRT in node-negative women with large tumors, and inclusion of internal mammary nodes. The final section discusses radiation field design and potential complications. (JNCCN 2007;5:331-338)
\end{abstract}

M treated with breast conservation therapy, including surgical tumor excision, radiation therapy, and adjuvant systemic therapy. However, mastectomy is still necessary for some women with breast cancer. Despite total mastectomy, these women still have a risk for locoregional failure (LRF) in the chest wall, skin, and regional lymph nodes. In certain cases, this risk warrants radiation to the

From the a Departments of Radiation Medicine and 'S ${ }^{\mathrm{S}}$ urgical Oncology, Roswell Park Cancer Institute, Buffalo, New York, and the 'Department of Surgery, University at Buffalo, Buffalo, New York.

Submitted October 9, 2006; accepted for publication October 24, 2006.

The authors have no financial interest, arrangement, or affiliation with the manufacturers of any products discussed in the article or their competitors.

Correspondence: Shaneli A. Fernando, MD, Roswell Park Cancer Institute, Elm and Carlton Streets, Buffalo, NY 14263. E-mail:

Shaneli.Fernando@roswellpark.org chest wall and regional lymphatics. Situations warranting post-mastectomy radiation (PMRT) include the finding of extensive nodal involvement, large tumor size, or positive margins. In other situations, the risk for LRF is low enough to omit radiation. These include cases with small cancers and negative nodes. Other situations are still debated, including cases with 1 to 3 positive lymph nodes, tumors larger than $5 \mathrm{~cm}$ with negative lymph nodes, and close surgical margins. This article reviews the role of PMRT based on the recommendations in the 2007 NCCN Breast Cancer Clinical Practice Guidelines in Oncology (see page 246). ${ }^{1}$

\section{Risk Factors for Locoregional Recurrence}

LRF occurs in some women despite total mastectomy. LRF is typically defined as cancer recurrence in the ipsilateral chest wall, including the scar and underlying muscle, and in the regional lymph nodes, including the supraclavicular, infraclavicular, axillary, and internal mammary nodes. Several groups have addressed risk factors that predict LRF by reviewing patterns of failure in women who underwent mastectomy without radiation. The Eastern Cooperative Oncology Group examined long-term risk for failure in more than 2000 women enrolled in 4 randomized trials conducted between 1978 and $1987 .{ }^{2}$ Patients in this series of trials underwent chemotherapy, mainly combination cyclophosphamide, methotrexate, and fluorouracil (CMF) with or without tamoxifen. The rate of LRF was higher among women with large tumors, negative hormone receptors, higher number of involved lymph nodes, and fewer lymph nodes resected. LRF at 10 years was $13 \%$ in women with 1 to 3 positive lymph nodes and $29 \%$ in women with 4 or more positive lymph nodes.

Larger tumor size and higher number of involved nodes are consistent factors associated with higher risk 
for LRF. ${ }^{2-7}$ Among 1000 women enrolled in doxorubicin-based chemotherapy studies at the M. D. Anderson Cancer Center, these factors affected the risk of LRF. 4.5 Additional risk factors that correlated with an increased risk for LRF included tumor extension beyond the lymph node capsule (i.e., extranodal extension), lymphovascular invasion, and gross multicentric disease.

The National Surgical Adjuvant Breast and Bowel Project (NSABP) reported on factors affecting LRF using pooled data from 5 randomized studies, including 5758 patients. ${ }^{6}$ LRF occurred in $25 \%$ of women with tumors $5 \mathrm{~cm}$ or larger compared with $15 \%$ of women whose primaries were less than $2 \mathrm{~cm}(P<.0001)$. LRF also correlated with the number of positive lymph nodes. Other factors in multivariate analysis that predicted LRF included premenopausal status, young age, and number of dissected lymph nodes.

The risk for LRF after mastectomy in women with negative nodes was examined in a series of almost 900 cases of women from the Massachusetts General Hospital. Several significant factors were associated with higher risk for LRF, including a surgical margin less than $2 \mathrm{~mm}$, tumors greater than $2 \mathrm{~cm}$, lymphovascular invasion, and premenopausal status. ${ }^{3}$

\section{PMRT Improves Local Control and Survival}

Several randomized trials and meta-analyses, discussed in detail below, show that PMRT reduces the risk for LRF by an average of two thirds. ${ }^{8-12}$ In addition to its effect on LRF, PMRT administered to women who also undergo appropriate adjuvant systemic therapy is increasingly being understood to positively impact on overall breast cancer survival. Clear evidence of this benefit was noted in the paired trials of the Danish Breast Cancer Collaborative Group (DBCCG). The DBCCG 82b trial randomized 1700 premenopausal women with stage II or III breast cancer treated with mastectomy to undergo either CMF chemotherapy for 9 cycles or 8 cycles of CMF with PMRT. ${ }^{13}$ All patients had positive lymph nodes, a tumor larger than $5 \mathrm{~cm}$, or invasion of skin or pectoral fascia. After 10 years of follow-up, LRF occurred in 9\% of women treated with surgery chemotherapy and PMRT compared with $32 \%$ of those treated with surgery and chemotherapy alone $(P<.001)$. In addition, PMRT significantly improved overall survival from $45 \%$ to $54 \%(P<.001)$.
The DBCCG 82c trial enrolled 1375 postmenopausal, stage II or III patients with breast cancer treated with mastectomy who had the same high-risk features as the $82 \mathrm{~b}$ study. ${ }^{8}$ After mastectomy, the study randomized women to treatment with tamoxifen alone or PMRT with tamoxifen. PMRT significantly reduced LRF ( $8 \%$ vs. $35 \%, P<.001)$ and improved 10 -year overall survival rate $(45 \%$ vs. $36 \% ; P=.03$ ).

The British Columbia Cancer Agency (BCCA) also conducted a randomized trial of PMRT, enrolling 318 premenopausal women with node-positive disease treated with mastectomy. ${ }^{9,10}$ These women underwent adjuvant chemotherapy and then were randomized to undergo radiation to the chest wall and regional lymphatics or no further therapy. Early followup through 15 years did not show significant differences in outcome. ${ }^{9}$ However, long-term follow-up with a median of 20.8 years showed significant differences in LRF and survival. ${ }^{10}$ Patients undergoing chemotherapy and PMRT had a 20-year overall survival of $47 \%$ compared with $37 \%$ for those who underwent chemotherapy alone $(P<.03)$, whereas breast cancer-specific survival showed an absolute improvement of $15 \%$ in women undergoing radiation (53\% vs. $38 \% ; P<.008$ ). Improvements in LRF (48\% vs. $30 \%$; $P<.001$ ) and distant metastatic-free survival (48\% vs. $31 \% ; P<.004)$ were also seen.

A recent meta-analysis by the Early Breast Cancer Trialists' Collaborative Group (EBCTCG) provided strong evidence supporting the use of PMRT. This group studied the results of 42,000 women treated in 78 randomized trials. ${ }^{12}$ Previous analyses with shorterterm follow-up showed a nonsignificant survival benefit in women undergoing radiation. A reduction in breast cancer mortality was offset by increased mortality from other causes. ${ }^{11}$ This increased mortality was largely attributed to vascular causes, particularly stroke that was independent of the laterality of the treated breast cancer. A 66\% reduction in LRF was also seen at 10 and 20 years, regardless of lymph node status. However, an update of the EBCTCG meta-analysis in 2005 with longer-term follow-up and more complete data showed a significant improvement in overall survival with PMRT for women with node-positive disease who underwent mastectomy, axillary dissection, and chemotherapy. ${ }^{12}$ The absolute improvement in survival was approximately $5 \%$. Another key finding was that a $20 \%$ reduction in 5-year local recurrence led to a $5 \%$ reduction in 15 -year breast cancer 
mortality (Figure 1). The EBCTCG concluded that radiation therapy is indicated to improve LRF and survival in situations where the reduction of LRF with radiation is estimated to be greater than $10 \%$.

Whelan et al. ${ }^{14}$ published results from another meta-analysis of patients with node-positive breast cancer enrolled in 18 trials examining the effects of locoregional radiation. Similar to the EBCTCG, this meta-analysis showed both a decrease in local recurrence (odds ratio [OR], $0.25 ; \mathrm{P}<.00001$ ) and mortality $(\mathrm{OR}, 0.83 ; \mathrm{P}=.004)$.

Importantly, the survival benefits of PMRT have been described in women who also undergo appropriate adjuvant systemic therapy. In cases requiring PMRT, this therapy is generally cytotoxic chemotherapy, with antiestrogen therapy added for those with hormone receptor-positive cancer. Chemotherapy and radiation are generally not administered concurrently because of increased rates of cutaneous reactions and possible increased effects on heart and lung tissue that limit the use of both treatments. The NCCN Breast Cancer Guidelines recommend that radiation be given at the conclusion of adjuvant chemotherapy. ${ }^{1}$

\section{Indications for PMRT}

Mastectomy alone is appropriate therapy in patients with small, node-negative tumors. Mastectomy may be necessary in patients with multicentric or active collagen vascular disease, prior chest radiation, or an anticipated or actual poor cosmetic outcome caused by the relationship of the tumor size or location to breast size, or because of patient choice after appropriate counseling. The rate of LRF for women who are node-negative with tumors less than $5 \mathrm{~cm}$ is low. However, some additional risk factors can be considered in this group. Jagsi et al. ${ }^{3}$ reviewed
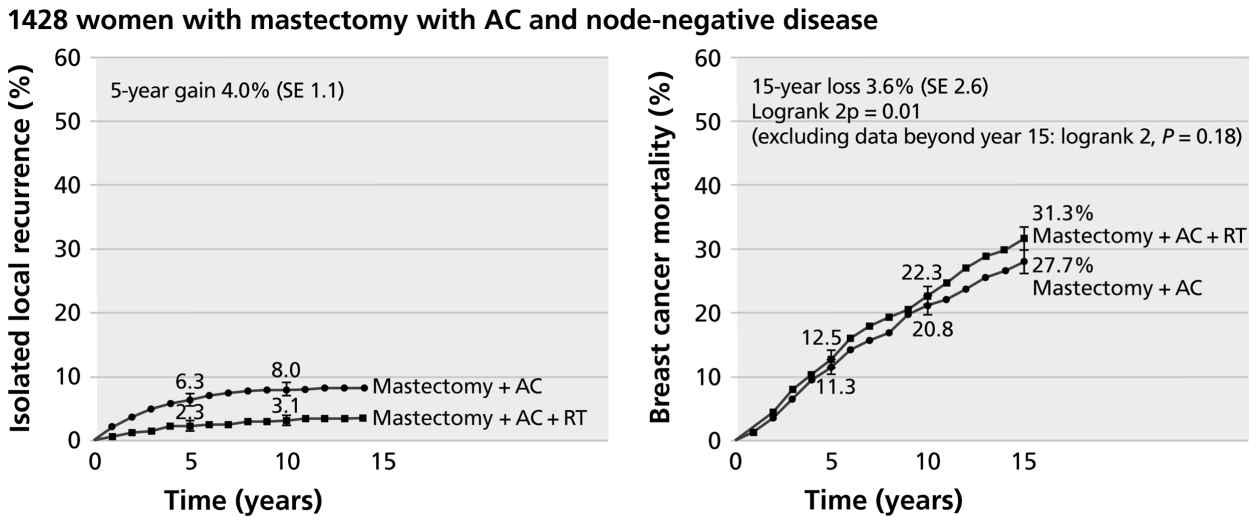

8505 women with mastectomy with $\mathrm{AC}$ and node-positive disease
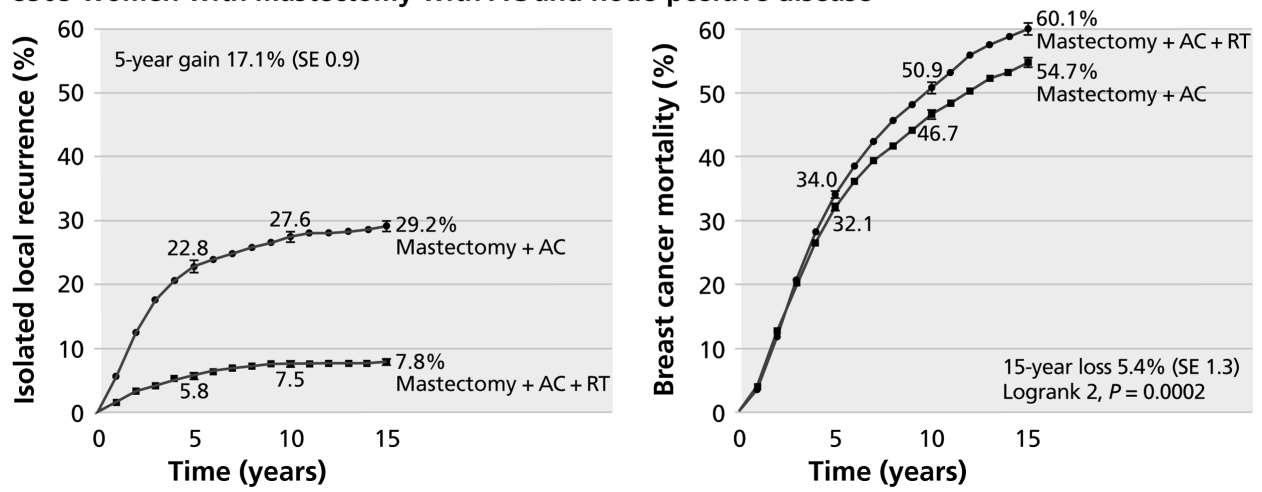

Figure 1 Effect of radiation and axillary clearance on local recurrence and breast cancer mortality.

Reprinted from The Lancet, 366, Clarke M, Collins R, Darby S, et al. Effects of radiotherapy and of differences in the extent of surgery for early breast cancer on local recurrence and 15-year survival: an overview of the randomized trials, 2087-2106, C2005, with permission from Elsevier.

Abbreviations: AC, axillary clearance; RT, radiation therapy; SE, standard error. 
Fernando and Edge

almost 900 cases of node-negative breast cancer treated with mastectomy without adjuvant radiation between 1980 and 2000. Several independent risk factors for LRF were present, including tumor size greater than $2 \mathrm{~cm}$, margin less than $2 \mathrm{~mm}$, premenopausal status, and LVI. As the number of risk factors increased, so did the rate of LRF in patients with no risk factors. These patients had a 10 -year LRF of 1\% compared with $40 \%$ in women with 3 risk factors. A study by the International Breast Cancer Study Group that included more than 1200 women with node-negative disease found increased risk of LRF in premenopausal women with vascular invasion and tumor larger than $2 \mathrm{~cm}$ and in post-menopausal women with vascular invasion. ${ }^{6}$ These studies suggest that additional risk factors should be considered in women with nodenegative disease with tumors smaller than $5 \mathrm{~cm}$. However, the benefit of PMRT in these women is less than $10 \%$ and does not meet the standard suggested by the EBCTCG. Omitting PMRT is appropriate for patients with negative nodes and a tumor smaller than $5 \mathrm{~cm}$ with negative margins. The rate of LRF in most series among these women is less than $10 \%$, and the benefit of PMRT does not meet the standard suggested by the EBCTCG. The NCCN guidelines advise against using PMRT in these situations. ${ }^{1}$

Conversely, the randomized control studies and meta-analyses discussed in the previous section show clear circumstances in which PMRT is indicated. As the extent of lymph node involvement in the axilla increases, so does the risk for LRF despite the use of adjuvant chemotherapy. ${ }^{8-10,13}$ The rate of LRF exceeds 20\% among women with extensive nodal involvement, typically classified as 4 or more lymph nodes, and those with skin or muscle involvement. Therefore, the NCCN guidelines recommend PMRT to the chest wall and supraclavicular nodes for patients who have 4 or more positive axillary lymph nodes, with consideration of radiation to the internal mammary lymph nodes. ${ }^{1}$

The value of PMRT for women with 1 to 3 positive lymph nodes and those with tumors larger than $5 \mathrm{~cm}$ without skin, muscle, or node involvement is of substantial debate. These women were included in the BCCA and DBCCG studies that showed a survival advantage for PMRT. Unplanned subset analyses of these studies did not identify a group in which PMRT had no benefit. Several key issues about these studies may have resulted in an unexpectedly high rate of LRF, including the extent of surgical dissection.
In the DBCCG studies, a median of 7 lymph nodes were removed. ${ }^{8,13}$ In general, the expected number of nodes is 10 or more with a standard level I to II dissection. This suggests that the axillary dissection in these studies was not complete, with a risk for nonresected, involved nodes. The rate of axillary failure was $13 \%$ in women who did not undergo radiation, which is significantly higher than the $1 \%$ to $3 \%$ rates of axillary failure observed in other series with complete axillary dissection. ${ }^{15}$ Therefore, the rate of LRF seen without PMRT in these studies may be artificially high and not reflect what is seen in current practice.

The benefit of PMRT with 1 to 3 positive nodes has been an area suitable for cooperative group trials given the intermediate risk for LRF, uncertainty of the benefit, and the retrospective nature of the existing data. In the late 1990s, an American cooperative group study was initiated to examine the role of postchemotherapy PMRT for women with tumors smaller than $5 \mathrm{~cm}$ who had 1 to 3 positive nodes. Unfortunately, the study failed to accrue the requisite number of patients and was closed with less than $10 \%$ of the planned sample size. The current National Cancer Institute of Canada (NCIC) MA-20 trial is enrolling women with 1 to 3 positive lymph nodes or high-risk, node-negative disease (tumor greater than $5 \mathrm{~cm}$, or greater than $2 \mathrm{~cm}$ with less than 10 lymph nodes examined) to whole breast or whole breast and regional nodal irradiation, including the internal mammary nodes.

The conflicting data on women with 1 to 3 positive nodes make recommendations less clear. The NCCN guidelines state that PMRT to the chest wall and supraclavicular lymph nodes should be considered for patients with 1 to 3 positive axillary lymph nodes. ${ }^{1}$

Data also conflict on the use of PMRT in women with tumors larger than $5 \mathrm{~cm}$ that do not involve the skin or underlying muscle and those with no associated lymph node metastasis (T3NOMO). Most series suggest that this category is associated with higher failure rates. ${ }^{4,6}$ Although T3NO cancers are not common, several series have examined recurrence patterns. Pathology was reviewed in a series of 81 patients initially believed to have T3NOMO breast cancer, and only 38 patients had accurate staging of T3NO.${ }^{16}$ Of 5 patients, 3 (60\%) who did not have PMRT had LRF compared with only 3 of 33 patients $(9 \%)$ who underwent PMRT $(P<.00003)$. The overall survival $(P$ $<.03)$ and disease-free survival rates $(P<.04)$ were also improved among patients undergoing PMRT. 
Use of Post-Mastectomy Radiation

However, other series have found lower rates of failure in patients with $\mathrm{T} 3 \mathrm{NO}$ disease, and the investigators recommended against PMRT in this situation. An NSABP study by Taghian et al. ${ }^{17}$ reviewed the results from more than 8800 women enrolled in 5 NSABP trials and found that 313 women had T3NO disease. Of these, 22 patients (7\%) experienced isolated LRF, with most patients experiencing recurrence at the chest wall. No significant factors associated with LRF were identified on multivariate analysis. A multi-institutional review by Floyd et al. ${ }^{18}$ that examined 70 cases of patients with T3, node-negative tumors found a 7.6\% LRF rate. Despite these conflicting data, the NCCN guidelines recommend radiation to the chest wall of patients with tumors greater than $5 \mathrm{~cm}$ with negative lymph nodes. ${ }^{1}$ The guidelines only advise that physicians consider, and do not recommend, radiation to the supraclavicular area and internal mammary lymph nodes in this setting.

The risk for LRF associated with a close or positive margin has also been an area of some controversy. A series from Freedman et al. ${ }^{19}$ reviewed pathology reports from almost 800 patients treated with mastectomy. They reported a close $(\leq 5 \mathrm{~mm})$ or positive margin in less than $5 \%$ of their patients with tumors smaller than $5 \mathrm{~cm}$ and 0 to 3 positive lymph nodes. Because subgroup analysis showed that patients younger than 50 years with close or positive margin had a $28 \%$ risk for LRF at 8 years even with chemotherapy, the authors recommended PMRT for these patients. The NCCN guidelines recommend chest wall radiation for patients with positive margins, with consideration of radiation to the supraclavicular and internal mammary lymph nodes.

\section{Radiation Targets}

LRF after mastectomy may occur in the skin of the chest wall, underlying muscle, and regional lymph nodes. Approximately $50 \%$ of LRFs occur at the chest wall, and therefore adequate coverage of the skin and mastectomy site is crucial. ${ }^{20}$ The next most common site of recurrence is the axillary apex, which includes the supraclavicular and infraclavicular lymph nodes and accounts for approximately one third of failures. As discussed previously, the addition of the supraclavicular field in patients with 1 to 3 lymph nodes is controversial. The BCCA and DBCCG randomized trials included the supraclavicular field and showed an improvement in survival, but whether this benefit would have been seen with radiation only to the chest wall is unknown. The NCCN guidelines recommend that experts include the supraclavicular field in women who have 4 or more positive nodes, consider a supraclavicular field in women with 1 to 3 lymph nodes if the chest wall is treated, and consider adding the supraclavicular field for patients with $\mathrm{T} 3$ primary and positive margins. ${ }^{1}$

Because isolated axillary failures are very low after a level I and II axillary dissection, the need for radiation to the axilla is unclear. The BCCA and DBCCG studies included the axilla in the field and showed an overall survival benefit. However, the extent of axillary dissection was less than optimal in these studies, with a median of only 7 and 11 lymph nodes, respectively, which probably accounted for the higher rates of axillary failure compared with other modern series. A study by Strom et al. ${ }^{21}$ from the M. D. Anderson Cancer Center examined more than 1000 women treated with mastectomy and level I/II dissection and chemotherapy without radiation therapy and found a 3\% recurrence rate in the low and mid axilla. The study notes several factors that increase the risk for LRF in the level I/II axillary apex region, including involvement of 4 or more lymph nodes, more than $20 \%$ nodal involvement, or gross extracapular extension. However, microscopic extracapsular extension does not seem to be associated with increased axillary failure. ${ }^{22}$ Given these low rates of axillary recurrence and the increased risk for lymphedema, routine axillary radiation is not recommended by the American Society of Clinical Oncology (ASCO) or NCCN. ${ }^{23}$

The other area at risk for regional lymph node involvement is the internal mammary nodes. No data clearly show that treatment of internal mammary nodes affects outcome, but this is debated among radiation oncologists. The fact that internal mammary nodes receive some dose from the standard, tangential fields used to treat the chest wall further complicates analysis of available data. The BCCA and DBCCG trials included internal mammary nodes and showed improved survival, but, similar to supraclavicular nodal inclusion, whether these areas specifically contributed to the improvement in outcomes is unknown. The NCCN guidelines recommend that experts consider internal mammary lymph node treatment when chest wall radiation is delivered. 


\section{Side Effects and Long-Term Morbidity from PMRT}

PMRT is generally well tolerated. The common, shortterm cutaneous effects seen with chest wall and nodal radiation are managed according to practice standards. Serious short- and long-term morbidity of PMRT is relatively uncommon. Long-term risks include radiation pneumonitis, heart damage, upper extremity lymphedema, brachial plexopathy, and secondary malignancies. In addition, radiation treatment in patients who have undergone breast reconstruction with tissue transfer or implant may be more difficult.

The risk for radiation pneumonitis increases with the volume of lung irradiated, which occurs when nodal irradiation is delivered in addition to chest wall radiation. ${ }^{24}$ Radiation pneumonitis is clinically characterized by low-grade fever, nonproductive cough, and infiltrate on chest radiograph, which typically develop several months after radiation therapy is completed. ${ }^{23}$ These side effects are self-limited in most patients, although some women may require steroids. Their incidence also increases with chemotherapy, especially with concurrent administration. A series from the Joint Center showed that rates were $9 \%$ with concurrent CMF chemotherapy versus $1 \%$ with sequential treatment. ${ }^{24}$ The concurrent use of taxanes, specifically weekly paclitaxel after adjuvant doxorubicin and cyclophosphamide, also resulted in increased rates of pneumonitis. ${ }^{26}$ The North Central Cancer treatment Group presented early results from the N9831 trial at ASCO 2006 for women treated with concurrent trastuzumab with breast or chest wall and nodal radiation, although internal mammary radiation was prohibited. No increase was seen in acute adverse events, including pneumonitis; long-term follow-up is required to assess late events. ${ }^{27}$

The effects of radiation on the heart can be difficult to assess given the long interval between treatment and most effects, notably ischemic heart disease. Other effects can include cardiomyopathy, valvular dysfunction, and pericarditis. The meta-analysis from the EBCTCG showed an increase in vascular mortality in PMRT patients. ${ }^{11,12}$ Some of these effects may be attributed to older techniques that treated larger volumes of heat than those included with computed tomography (CT)-based planning today. A modern Joint Center series showed no difference in cardiac events in right- and left-sided breast conservation patients with a follow-up of 12 years. ${ }^{28}$ A recently published series from the University of Pennsylvania showed an increased rate of myocardial infarction and coronary artery disease in patients with left-sided breast cancer who underwent breast or chest wall radiation when compared with those with right-sided cancer undergoing similar treatment, but this was not associated with a higher risk for cardiac death up to 20 years after radiation treatment. ${ }^{29} \mathrm{~A}$ concern also exists that patients treated with doxorubicin-containing regimens, taxanes, or trastuzumab may be at higher risk for long-term cardiac morbidity when also undergoing PMRT. Care should be taken in radiation planning to minimize the dose to the myocardium. This is especially important if radiation fields are designed to include the internal mammary nodes.

Lymphedema results from damage to the lymphatic drainage system of the upper extremity in the axilla. This can develop after axillary dissection without radiation or with radiation alone. Combined axillary dissection and radiation increases the risk for lymphedema. A series from the Joint Center involving patients who underwent full axillary dissection and axillary radiation showed a $36 \%$ risk compared with $6 \%$ for women who underwent axillary radiation after a limited dissection. ${ }^{30}$ Data from the Roswell Park Cancer Institute found several factors associated with increased risk for lymphedema, including higher total dose of radiation, use of posterior axillary boost, and overlap of radiation fields. ${ }^{31}$ As discussed previously, additional axillary radiation is not recommended in women who have undergone adequate axillary surgery, because rates of axillary recurrence are low. Omitting additional axillary radiation when using the supraclavicular field to include the apex of the axilla (level III) may minimize risk for lymphedema.

Brachial plexopathy is an uncommon effect of PMRT that has been associated with increasing doses of radiation, typically more than $50 \mathrm{~Gy} .{ }^{32}$ The $5 \%$ risk for injury at 5 years has been estimated to be a dose of 60 Gy to the entire plexus, whereas the $50 \%$ risk of plexopathy at 5 years is estimated at $75 \mathrm{~Gy} .{ }^{33}$ Because this is typically much higher than doses given to patients with CT-based planning, brachial plexopathy is uncommon.

Another rare but serious complication of radiation is the risk for developing a radiation-induced malignancy. The most common type of malignancy is angiosarcoma, which typically develops 6 to 10 years after treatment. In a large series by the Institut 
Use of Post-Mastectomy Radiation

Gustav-Roussy, 9 of 7620 patients developed sarcoma with a 10 -year actuarial incidence of $0.2 \%, 20$-year incidence of $0.43 \%$, and 30 -year incidence of $0.78 \% .{ }^{34}$ The second type of malignancy most often associated with the radiation field is lung cancer. A case control study from the Connecticut Tumor Registry showed a small elevation in ipsilateral lung cancers in women treated more than 10 years previously for breast cancer. ${ }^{35}$

Because many patients desire breast reconstruction after mastectomy, the type of reconstruction and optimal sequence in relation to radiation therapy becomes an issue. Complication rates are lower for irradiated autologous reconstructions compared with expander-implants. A study from the Massachusetts General Hospital found a 53\% complication rate at 2 years in 18 patients undergoing expander-implant reconstructions compared with $12 \%$ in 30 patients undergoing transverse rectus abdominus myocutaneous flap. ${ }^{36}$ Implant failure rates can be as high as $37 \%$ for patients undergoing expander-implant reconstruction before radiation. ${ }^{37}$ Implant failure is lower in women who complete PMRT before reconstruction. ${ }^{38}$ Concerns also exist that immediate breast reconstruction may adversely affect radiation treatment planning. A study of 110 patients from the M. D. Anderson Cancer Center compared treatment plans of patients who underwent mastectomy with immediate reconstruction and radiation to stage-matched control undergoing PMRT without reconstruction..$^{39}$ They found compromise in $52 \%$ of plans in the immediate reconstruction group compared with $7 \%$ in the control group. If a patient is likely to need PMRT, delayed reconstruction should be considered.

\section{Conclusions}

The NCCN guidelines recommend the use of PMRT for patients with 4 or more positive axillary lymph nodes, $5 \mathrm{~cm}$ or larger lesions, and a positive margin after mastectomy. Some areas of controversy exist and have been incorporated into the guidelines, including treatment of women with 1 to 3 positive axillary lymph nodes and inclusion of internal mammary lymph nodes in target volumes. With newer imaging and planning technology, the morbidity associated with PMRT has decreased. However, experts must be aware of the interactions of systemic therapy with PMRT, specifically regarding heart and lung toxicity. Finally, many questions remain about indications, targets, and toxicity of PMRT. Clinicians are strongly encouraged to participate in any available clinical trial designed to address these questions.

\section{References}

1. Carlson RW, Anderson BO, Burstein HJ, et al. NCCN breast cancer clinical practice guidelines in oncology. Version 1, 2007. Available at: http://www.nccn.org/physician_gls/PDF/breast.pdf. Accessed January 25, 2007.

2. Recht $A$, Gray R, Davidson NE, et al. Locoregional failure 10 years after mastectomy and adjuvant chemotherapy with or without tamoxifen without irradiation: experience of the Eastern Cooperative Oncology Group. J Clin Oncol 1999;17:1689-1700.

3. Jagsi R, Raad RA, Goldberg S, et al. Locoregional recurrence rates and prognostic factors for failure in node-negative patients treated with mastectomy: implications for postmastectomy radiation. Int J Radiat Oncol Biol Phys 2005;62:1035-1039.

4. Katz A, Strom EA, Buchholz TA, et al. Locoregional recurrence patterns after mastectomy and doxorubicin-based chemotherapy: implications for postoperative irradiation. J Clin Oncol 2000;18: 2817-2827.

5. Katz A, Strom EA, Buchholz TA, et al. The influence of pathologic tumor characteristics on locoregional recurrence rates following mastectomy. Int J Radiat Oncol Biol Phys 2001;50:735-742.

6. Taghian A, Jeong JH, Mamounas E, et al. Patterns of locoregional failure in patients with operable breast cancer treated by mastectomy and adjuvant chemotherapy with or without tamoxifen and without radiotherapy: results from five National Surgical Adjuvant Breast and Bowel Project randomized clinical trials. J Clin Oncol 2004;22:4247-4254.

7. Wallgren A, Bonetti M, Gelber RD, et al. Risk factors for locoregional recurrence among breast cancer patients: results from international breast cancer study group trials I through VII. J Clin Oncol 2003;21:1205-1213.

8. Overgaard M, Jensen MB, Overgaard J, et al. Postoperative radiotherapy in high risk postmenopausal breast cancer patients given adjuvant tamoxifen: Danish Breast Cancer Cooperative Group DBCG 82c randomized trial. Lancet 1999;353:1641-1648.

9. Ragaz J, Jackson SM, Le N, et al. Adjuvant radiotherapy and chemotherapy in node-positive premenopausal women with breast cancer. N Engl J Med 1997;337:936-962.

10. Ragaz J, Olivotto IA, Spinell JJ, et al. Locoregional radiation therapy in patients with high-risk breast cancer receiving adjuvant chemotherapy: 20-year results of the British Columbia randomized trial. J Natl Cancer Inst 2005;97:116-126.

11. Favourable and unfavourable effects on long-term survival of radiotherapy for early breast cancer: an overview of the randomized trials. Early Breast Cancer Trialists' Collaborative Group. Lancet 2000;355:1757-1770.

12. Clarke M, Collins R, Darby C, et al. Effects of radiotherapy and of differences in the extent of surgery for early breast cancer on local recurrence and 15-year survival: an overview of the randomized trials. Lancet 2005;366:2087-2106.

13. Overgaard M, Hansen PS, Overgaard J, et al. Postoperative radiotherapy in high-risk premenopausal women with breast cancer who receive adjuvant chemotherapy. N Engl J Med 1997;337:949-955.

14. Whelan TJ, Julian J, Wright J, et al. Does locoregional radiation therapy improve survival in breast cancer? A meta-analysis. J Clin Oncol 2000;18:1220-1229. 
Fernando and Edge

15. Benson EA, Thorogood J. The effect of surgical technique on local recurrence rates following mastectomy. Eur J Surg Onc 1986;12: 267-271.

16. $\overline{\text { Helinto } M}$, Blomqvist C, Heikkila P, Joensuu H. Post-mastectomy radiotherapy in pT3NOMO breast cancer: is it needed? Radiother Oncol 1999;52:213-217.

17. Taghian AG, Jeong JH, Manmouas EP, et al. Low locoregional recurrence rate among node-negative breast cancer patients with tumors $5 \mathrm{~cm}$ or larger treated by mastectomy, with or without adjuvant systemic therapy and without radiotherapy: results from five National Surgical Adjuvant Breast and Bowel Project randomized clinical trials. J Clin Oncol 2006;24:3927-3932.

18. Floyd, SR, Buchholz TA, Haffty BG, et al. Low local recurrence rate without postmastectomy radiation in node-negative breast cancer patients with tumors $5 \mathrm{~cm}$ and larger. Int J Radiat Oncol Biol Phys 2006;66:358-364.

19. Freedman GM, Fowble BL, Hanlon AL, et al. A close or positive margin after mastectomy is not an indication for chest wall irradiation except in women aged fifty or younger. Int J Radiat Oncol Biol Phys 1998;41:599-605.

20. Strom EA, McNeese MD. Postmastectomy irradiation rationale for treatment field selection. Semin Radiat Oncol 1999;9:247-253.

21. Strom EA, Woodward WA, Katz A, et al. Clinical investigation: regional nodal failure patterns in breast cancer patients treated with mastectomy without radiotherapy. Int J Radiat Oncol Biol Phys 2005;63:1508-1513.

22. Pierce LJ, Oberman HA, Strawderman MH, Lichter AS. Microscopic extracapsular extension in the axilla: is this an indication for axillary radiotherapy? Int J Radiat Oncol Biol Phys 1995;33:253-259.

23. Rech A, Edge SB, Solin LJ, et al. Postmastectomy radiotherapy: guidelines for the American Society of Clinical Oncology. J Clin Oncol 2001;19:1589-1569.

24. Lingos TI, Recht A, Vacini F. Radiation pneumonitis in breast cancer patients treated with conservative surgery and radiation therapy. Int J Radiat Oncol Biol Phys 1991;21:355-360.

25. Marks LB. Pulmonary effects of thoracic irradiation. Oncology 1994;8:89-100.

26. Burstein HJ, Bellon JR, Galper S, et al. Prospective evaluation of concurrent paclitaxel and radiation therapy after adjuvant doxorubicin and cyclophosphamide chemotherapy for stage II or III breast cancer. Int J Radiat Oncol Biol Phys 2006;64:496-504.
27. Halyard M, Pisansky L, Solin U, et al. Adjuvant radiotherapy (RT) and trastuzumab in stage I-IIA breast cancer; toxicity data from the North Central Cancer Treatment Group Phase III trial N9831. J Clin Oncol 2006;24(suppl 1):8S. Abstract 523.

28. Nixon AJ, Manola J, Gelman R. No long term increase in cardiacrelated mortality after breast conserving surgery and radiation using modern techniques. J Clin Oncol 1998;16:1374-1379.

29. Harris EE, Correa C, Hwang WT, et al. Late cardiac mortality and morbidity in early stage breast cancer patients after breast conservation treatment. J Clin Oncol 2006;24:4100-4106.

30. Larson D, Weinstein M, Goldberg I. Edema of the arm as a function of the extent of axillary surgery in patient with stage I-II carcinoma of the breast treated with primary radiotherapy. Int J Radiat Oncol Biol Phys 1986;12:1575-1582.

31. Hinrichs CS, Watroba NL, Rezaishiraz H, et al. Lymphedema secondary to post-mastectomy radiation: incidence and risk factors. Ann Surg Oncol 2004;11:573-580.

32. Yeoh EK, Denham JW, Davies SA, Spittle MF. Primary breast cancer. Complications of axillary management. Acta Radiol Oncol $1986 ; 25: 105-108$

33. Emami B, Lyman J, Brown A, et al. Tolerance of normal tissues to therapeutic irradiation. Int J Radiat Oncol Biol Phys 1991;21:109-122.

34. Taghian $\mathrm{A}$, de Vathaire $\mathrm{F}$, Terrier $\mathrm{P}$, et al. Long-term risk of sarcoma following radiation treatment for breast cancer. Int J Radiat Oncol Biol Phys 1991;21:361-367.

35. Neugut AI, Robinson E, Lee WC, et al. Lung cancer after radiation therapy for breast cancer. Cancer 1993;71:3054-3057.

36. Chawla AK, Kachnic LA, Taghian AG, et al. Radiotherapy and breast reconstruction: complication and cosmesis with TRAM versus tissue expander/ implant. Int J Radiat Oncol Biol Phys 2002;54:520-526.

37. Krueger EA, Wilkinson EG, Strawderman MH, et al. Complications and patient satisfaction following expander/ implant breast reconstruction with and without radiotherapy. Int J Radiat Oncol Biol Phys 2001;49:713-721.

38. Ascherman JA, Hanasono MM, Newman MI, Hughes DB. Implant reconstruction in breast cancer patients treated with radiation therapy. Plast Reconstr Surg 2006;117:359-365.

39. Motwani SB, Strom EA, Schechter NR, et al. The impact of immediate breast reconstruction on the technical delivery of postmastectomy radiotherapy. Int J Radiat Oncol Biol Phys 2006;66:76-82. 